\title{
Visibilidad Científica de las Universidades Colombianas en ResearchGate
}

\author{
Vanessa P. Pertuz, Adith B. Pérez y Adriana L. Vega \\ Universidad de Santander, Facultad de Ingenierías, Grupo de Investigación Nuevas Tecnologías-UDES, \\ Valledupar, Colombia (e-mail: van.pertuz@mail.udes.edu.co; adi.perez@mail.udes.edu.co; \\ val13151008@mail.udes.edu.co)
}

Recibido Abr. 25, 2018; Aceptado Jun. 29, 2018; Versión final Jul. 20, 2018, Publicado Dic. 2018

\begin{abstract}
Resumen
El artículo compara la visibilidad científica de las universidades colombianas a partir del indicador de ResearchGate (RGScore). El artículo analiza las 150 universidades colombianas que cuenta con perfil en ResearchGate de las 305 registradas en el Sistema Nacional de Información de la Educación Superior (SNIES). Las 10 universidades con mayor RGScore son: Universidad Nacional de Colombia, Universidad de Antioquia, Universidad de Los Andes, Universidad del Valle, Pontificia Universidad Javeriana, Universidad Industrial de Santander, Universidad del Norte, Universidad de Cartagena, Universidad de la Sabana y Universidad Pontificia Bolivariana; instituciones acreditadas en Alta Calidad por el estado colombiano. Adicionalmente, existe una correlación positiva débil entre el número de Grupos de Investigación, Desarrollo Tecnológico e Innovación clasificados y reconocidos por Colciencias por departamento y promedio RGScore de las Universidades por Departamento en Colombia.
\end{abstract}

Palabras clave: ResearchGate; visibilidad universidades colombianas; RGScore

\section{Scientific Visibility of Colombian Universities in ResearchGate}

\begin{abstract}
The paper aims to compare the scientific visibility of Colombian universities based on the ResearchGate indicator (RGScore). The article analyzes the 150 Colombian universities that have a ResearchGate profile of the 305 registered in the National Information System of Higher Education (SNIES). The top 10 universities with the best positions of RGScore are: Universidad Nacional de Colombia, Universidad de Antioquia, Universidad de Los Andes, Universidad del Valle, Pontificia Universidad Javeriana, Universidad Industrial de Santander, Universidad del Norte, Universidad de Cartagena, Universidad de la Sabana y Universidad Pontificia Bolivariana. These are institutions accredited in High Quality by the Colombian government. Additionally, there is a weak positive correlation between the number of Groups of Research, Technological Development and Innovation classified and recognized by Colciencias per regions and average RGScore of the Universities in each Colombian Department.
\end{abstract}

Keywords: ResearchGate; visibility colombian universities; RGScore 


\section{INTRODUCCIÓN}

Las redes sociales orientadas a la difusión de la información científica corresponden a un elemento fundamental en el contexto actual, dado el desarrollo de la Web 2.0 (Santana, 2010). En este sentido, los autores Puentes et al. (2015) señalan que las redes sociales se han convertido, en poco tiempo, en la mayor expresión de interactividad, permitiendo la colaboración de investigadores a escala mundial y el intercambio de información científica (Ortega, 2017). Específicamente, ResearchGate es una red de investigadores que permite compartir información. De acuerdo con lo planteado por los autores Orduña et al. (2016), ResearchGate es una de las más importantes redes sociales académicas, con más de 9.000 .000 de usuarios y 80.000.000 de documentos. Adicionalmente, los autores en referencia destacan como un valor agregado de ResearchGate un amplio catálogo de indicadores bibliométricos, entre los que se encuentra el RGScore. En este sentido, Yu et al. (2016) destacan que, algunas métricas específicas, como el puntaje RG (RGScore) de ResearchGate, que integra tanto bibliometría como altmetría, han sido reconocidas como un indicador efectivo para medir el desempeño tanto institucional como individual. De la misma manera, Aguillo et al. (2017) concluyen que tanto Google Scholar Citations (GSC) como ResearchGate (RG) tienen una tendencia bibliométrica, aunque en el caso de ResearchGate, existen también componentes relacionados con las prácticas de acceso abierto y el uso de indicadores altmétricos.

Así, los autores Singson y Amees (2017) destacan la amplia utilización de ResearchGate en los investigadores de la Universidad de Pondicherry; especialmente, para conectarse con personas que tienen intereses similares. En efecto, Bhardwaj (2017) evalúa las redes ResearchGate, Academia.edu, Mendeley y Zotero, destacando que ResearchGate obtiene la mayor puntuación. En consecuencia, una revisión de literatura desarrollada por Manca (2018) destaca que la cantidad de estudios centrados en ResearchGate es más del doble que los dedicados a Academia.edu.

De la misma manera, Muscanell y Utz (2017) analizan el uso y los beneficios de ResearchGate en investigadores de Europa y Estados Unidos. Mientras que Míguez et al. (2017) analizan estos aspectos en las Universidades de Portugal; y Araujo et al. (2017) en el contexto de las comunidades científicas brasileñas y portuguesas. En el mismo orden de ideas, Jamali (2017) analiza si los miembros de ResearchGate, como autores de artículos de revistas cumplen con las políticas de derechos de autor de las editoriales. Paralelamente, el estudio de los autores Puentes et al. (2015) señalan que, a pesar de los múltiples beneficios de esta red de investigación, el número de investigadores conscientes de estos beneficios y dispuestos a aprovecharlos aún es insuficiente. El trabajo de los autores antes mencionados, realizado en el contexto de las universidades de Portugal, destaca que solo el $49.5 \%$ del personal académico de las universidades analizadas está registrado en ResearchGate o Academia.edu. En este sentido, "en un contexto en el que la colaboración se está convirtiendo en el mejor método de producción informativa e investigadora, ignorar este tipo de plataformas es un camino erróneo" (Puentes et al., 2015, p.2845).

En concordancia con lo anterior, un estudio realizado por González et al. (2015), orientado a estudiar la presencia de 77 universidades españolas en las redes científicas ResearchGate y Academia.edu, evidencia que, pese al desarrollo de estas redes de investigación, el número de miembros españoles en estas redes sociales es insuficiente y en algunas universidades "casi anecdótico". Por su parte, investigaciones desarrolladas en el contexto latinoamericano, puntualmente referentes a presencia e impacto en Researchgate.net y Academia.edu de 165 universidades de los cuatro países andinos (Colombia, Perú, Ecuador y Bolivia), se concluye que la presencia e impacto de las universidades andinas en estas redes es desigual, aunque emergente y creciente, pero aún divergente con respecto a los resultados registrados en otros rankings universitarios internacionales de más amplia tradición (Campos Freire et al., 2014). De esta manera, ResearchGate, mediante su indicador de reputación (RGScore), registra el impacto y popularidad, es decir, producción e interacción del investigador ante sus colegas (Punín et al., 2014); mientras que el índice $\mathrm{H}$ de Google mide impacto y productividad. Así, a diferencia de las métricas tradicionales, el RGScore se centra en el investigador y es mucho más dinámico, porque a través de la conversación y la interacción científica se va autogenerando (Campos et al., 2014). Bajo este contexto, se van consolidando las métricas alternativas, correspondientes a una serie de indicadores que miden el impacto de la investigación, cuantificando su presencia en la web social (Galligan y Dyas, 2013); (Robinson et al., 2014), como herramienta complementaria al método tradicional de citas en los JCR (ISI) y SJR (Scimago).

De otro lado, los autores Mangkhollen y Mohammad (2017) señalan que la puntuación métrica de ResearchGate ha sido objeto de numerosos estudios. Si bien, la métrica ResearchGate aún no se ha establecido como una métrica de evaluación de credibilidad entre la comunidad académica, está ganando impulso al reinventarse a sí misma como una medida de impacto social. En el mismo sentido, Thelwall y Kousha (2017) señalan que ResearchGate proporciona una calificación general para cada miembro académico, por lo que el puntaje RGScore, refleja una combinación de logros académicos y actividades dentro del sitio (Orduña et al., 2016), no obstante, el RGScore se correlaciona razonablemente bien con otros 
indicadores de prestigio académico para investigadores (Yu et al., 2016). Por su parte, las investigaciones de los autores Orduña et al. (2016) establecen que el indicador RGScore, no mide el prestigio de los investigadores sino su nivel de participación en la plataforma. De la misma, manera el estudio de Orduna et al. (2017) afirma que los altos puntajes de RGScore se basan principalmente en la actividad relacionada con preguntas y respuestas en el sitio.

Considerando lo anterior, el presente artículo analiza la visibilidad de las universidades colombianas con base en el indicador RGScore y su relación con otros indicadores de calidad, productividad y ubicación geográfica. Como limitaciones del estudio, se debe tener en cuenta que los indicadores de bibliometría y altmetría son dinámicos y dependen de la actividad científica en la web. Estos estudios varían de acuerdo al momento y a las circunstancias en las cuales se recogen los datos. Por ejemplo, los indicadores de ResearchGate se modifican semanalmente, mientras los indicadores del sistema de ciencia y tecnología de Colciencias se actualizan cuando se realizan convocatorias, en este caso cada 2 años. Adicionalmente, metodológicamente, en la presente investigación se estima la relación entre el número de grupos de investigación, desarrollo tecnológico e innovación en Colombia (laboratorios de investigación, en otros países); su distribución geográfica y el promedio RGScore de las Universidades por departamento en Colombia.

En cuanto a futuras investigaciones es posible incluir otras métricas de redes de investigación, análisis de redes sociales y realizar una comparación con los indicadores de medición y clasificación de investigadores de cada país, con el fin de determinar hasta qué punto las redes de investigación se corresponden con la calidad y visibilidad de la producción de los investigadores y de las universidades. De igual manera, se sugiere seleccionar muestras del RGScore individual de los investigadores por Universidad, con el objeto de analizar la productividad, el impacto, la reputación y la visibilidad de las publicaciones en ResearchGate. Además, es posible incluir la realización de estudios longitudinales de la temática debido a la dinámica de la actividad científica en las bases de datos indexadas y las redes sociales, así como análisis de redes sociales para estudiar a profundidad las relaciones de cooperación que establecen las universidades colombianas.

\section{METODOLOGÍA}

La presente investigación es de tipo descriptivo (Tamayo, 2011) con un diseño no experimental y transversal (Hernández et al., 2014). En Colombia el Sistema Nacional de Información de la Educación Superior (SNIES) registra los datos concernientes a las universidades. EI SNIES indica que actualmente existen 305 universidades activas en Colombia de las cuales 150 cuentan con perfiles registrados en ResearchGate (2017). El presente trabajo considera los indicadores definidos en la tabla 1.

Tabla 1: Definición conceptual y matemática de los indicadores

\begin{tabular}{|c|c|c|}
\hline $\begin{array}{l}\text { Nombre } \\
\text { indicador. }\end{array}$ & Definición conceptual. & $\begin{array}{l}\text { Definición matemática / Fuente de } \\
\text { información. }\end{array}$ \\
\hline $\begin{array}{l}\text { Acreditación } \\
\text { alta calidad. }\end{array}$ & $\begin{array}{l}\text { Reconocimiento del estado colombiano en torno } \\
\text { a la calidad de los programas académicos de una } \\
\text { institución, su organización, funcionamiento y el } \\
\text { cumplimiento de su función social. }\end{array}$ & $\begin{array}{l}\text { Indicador definido de la información del } \\
\text { Consejo Nacional de Acreditación de } \\
\text { Colombia (2017) }\end{array}$ \\
\hline $\begin{array}{l}\text { Ubicación } \\
\text { geográfica. }\end{array}$ & $\begin{array}{l}\text { Municipio y departamento (División } \\
\text { administrativa y política) en el que se ubica la } \\
\text { sede principal de la Universidad. }\end{array}$ & $\begin{array}{l}\text { Tomado de la base de datos del Sistemá } \\
\text { Nacional de Información de la Educación } \\
\text { Superior (SNIES) (2017). }\end{array}$ \\
\hline $\begin{array}{l}\text { Número total de } \\
\text { miembros } \\
\text { registrados en } \\
\text { ResearchGate. }\end{array}$ & $\begin{array}{l}\text { Número de investigadores que se encuentran } \\
\text { registrados en el perfil de ResearchGate de cada } \\
\text { universidad. }\end{array}$ & $\begin{array}{l}\text { Tomado del perfil de las universidades en } \\
\text { ResearchGate. }\end{array}$ \\
\hline $\begin{array}{l}\text { Total RGScore de } \\
\text { la universidad. }\end{array}$ & $\begin{array}{l}\text { Medida de la reputación científica de las } \\
\text { instituciones, en función de cómo la investigación } \\
\text { de cada uno de los investigadores es percibida } \\
\text { por sus pares (Orduña et al., 2016). }\end{array}$ & $\begin{array}{l}\text { RGScore corresponde al cómputo de número } \\
\text { de publicaciones y puntos totales por perfiles } \\
\text { adscritos a una universidad, así como los } \\
\text { puntos globales adscritos a una disciplina de } \\
\text { conocimiento concreto (González et al., } \\
\text { 2015). Los tres componentes que se tienen } \\
\text { en cuenta para su cálculo son: las } \\
\text { contribuciones cargadas por el autor; } \\
\text { interacciones con otros miembros; y la } \\
\text { reputación adquirida de otros investigadores } \\
\text { (Orduña et al., 2016). No obstante, la } \\
\text { composición exacta de estos tres } \\
\text { componentes no es publicada, al igual que su } \\
\text { ponderación (Orduna et al., 2017). }\end{array}$ \\
\hline
\end{tabular}


Tabla 1 (continuación)

\begin{tabular}{|c|c|c|}
\hline $\begin{array}{l}\text { Total de } \\
\text { publicaciones } \\
\text { registradas en } \\
\text { ResearchGate. }\end{array}$ & $\begin{array}{l}\text { Número total de publicaciones (por ejemplo, } \\
\text { artículos, presentaciones, informes, documentos } \\
\text { de trabajo, datos en bruto); que se encuentran } \\
\text { registrados en el perfil de cada uno de los } \\
\text { miembros de las universidades en } \\
\text { ResearchGate. }\end{array}$ & Perfil de las universidades en ResearchGate. \\
\hline $\begin{array}{l}\text { Principales } \\
\text { Instituciones } \\
\text { Colaboradoras. }\end{array}$ & $\begin{array}{l}\text { Colaboración de otras instituciones con los } \\
\text { investigadores registrados en cada institución. }\end{array}$ & Perfil de las universidades en ResearchGate. \\
\hline $\begin{array}{lr}\text { Número } & \text { de } \\
\text { Grupos } & \text { de } \\
\text { Investigación, } & \\
\text { Desarrollo } & \\
\text { Tecnológico } & \text { e } \\
\text { Innovación } & \\
\text { clasificados } & \text { y } \\
\text { reconocidos } & \text { por } \\
\text { Colciencias } & \text { por } \\
\text { departamento. } & \end{array}$ & $\begin{array}{l}\text { Se entiende como Grupo de Investigación, } \\
\text { Desarrollo Tecnológico e Innovación "al conjunto } \\
\text { de personar que interactúan para investigar y } \\
\text { generar productos de conocimiento de uno o } \\
\text { varios temas, de acuerdo con un plan de trabajo } \\
\text { corto, mediano o largo plazo (tendiente a la } \\
\text { solución de un problema)". Un grupo es } \\
\text { reconocido como tal, siempre que demuestre } \\
\text { continuamente resultados verificables, derivados } \\
\text { de proyectos y de otras actividades procedentes } \\
\text { de su plan de trabajo y que además cumpla con } \\
\text { los requisitos mínimos para su reconocimiento. } \\
\text { (Departamento Administrativo de Ciencia, } \\
\text { Tecnología e Innovación - COLCIENCIAS, 2017). } \\
\text { En otros paises habitualmente se denominan } \\
\text { laboratorios de investigación. }\end{array}$ & $\begin{array}{lll}\text { Plataforma SCIENTI } & \text { (Departamento } \\
\text { Administrativo de Ciencia, Tecnología e } \\
\text { Innovación - COLCIENCIAS, 2017). }\end{array}$ \\
\hline $\begin{array}{l}\text { Promedio } \\
\text { RGScore de las } \\
\text { Universidades por } \\
\text { departamento. }\end{array}$ & $\begin{array}{l}\text { Determinar la visibilidad relativa en } \\
\text { ResearchGate por cada departamento. }\end{array}$ & $\begin{array}{l}\text { Se hace la sumatoria de los RGScore de cada } \\
\text { una de las instituciones con sede principal en } \\
\text { cada departamento y se divide entre el } \\
\text { número total de instituciones de cada uno de } \\
\text { los departamentos. }\end{array}$ \\
\hline $\begin{array}{lr}\text { Ranking Top } & 10 \\
\text { RGScore de las } \\
\text { universidades } \\
\text { acreditadas } \\
\text { Colombia. }\end{array}$ & $\begin{array}{l}\text { Ranking de las universidades acreditadas con } \\
\text { mayor puntaje RGScore. }\end{array}$ & $\begin{array}{l}\text { Se seleccionan las } 10 \text { universidades } \\
\text { acreditadas de Colombia con mayor } \\
\text { RGScore. }\end{array}$ \\
\hline $\begin{array}{lr}\text { Ranking Top } & 10 \\
\text { RGScore de las } \\
\text { universidades } & \text { no } \\
\text { acreditadas } & \text { en } \\
\text { Colombia. } & \\
\end{array}$ & $\begin{array}{l}\text { Ranking de las universidades no acreditadas con } \\
\text { mayor puntaje RGScore. }\end{array}$ & $\begin{array}{l}\text { Se seleccionan las } 10 \text { universidades no } \\
\text { acreditadas de Colombia con mayor } \\
\text { RGScore. }\end{array}$ \\
\hline $\begin{array}{l}\text { Ranking Top } 10 \\
\text { RGScore de las } \\
\text { universidades de } \\
\text { Bogotá. }\end{array}$ & $\begin{array}{l}\text { Ranking de las universidades localizadas en la } \\
\text { ciudad de Bogotá, Colombia con mayor puntaje } \\
\text { RGScore. }\end{array}$ & $\begin{array}{l}\text { Se seleccionan las } 10 \text { universidades } \\
\text { localizadas en Bogotá con mayor RGScore. }\end{array}$ \\
\hline $\begin{array}{l}\text { Ranking Top } 10 \\
\text { RGScore de las } \\
\text { universidades de } \\
\text { Colombia excepto } \\
\text { Bogotá. }\end{array}$ & $\begin{array}{l}\text { Ranking de las universidades de Colombia } \\
\text { exceptuando las localizadas en la ciudad de } \\
\text { Bogotá, con mayor puntaje RGScore. }\end{array}$ & $\begin{array}{l}\text { Se seleccionan las } 10 \text { universidades de } \\
\text { Colombia con mayor RGScore, exceptuando } \\
\text { las localizadas en la ciudad de Bogotá. }\end{array}$ \\
\hline
\end{tabular}

Para efectos de la recolección de los datos correspondientes a los indicadores objeto de estudio, se consultaron en la página de ResearchGate cada uno de los perfiles de las Universidades registradas en el SNIES. Los datos para el cálculo de los indicadores fueron tomados la última semana del mes de noviembre del año 2017. El análisis de los datos se realiza mediante técnicas de estadística descriptiva e inferencial.

\section{RESULTADOS}

A continuación, se presentan los resultados del análisis de los indicadores considerados en el artículo. La tabla 2 presenta las universidades colombianas con mayor puntaje en el indicador total de RGScore. Para efectos de este análisis, se consideraron las universidades Colombianas registradas en la base de datos del Sistema Nacional de Información de la Educación Superior (SNIES) (2017). La clasificación de estas universidades se realiza, en orden descendente, en función del Total RGScore de la Universidad. Los resultados de la Tabla 2 indican que las 10 universidades con mejor posicionamiento en ResearchGate son, respectivamente: Universidad Nacional de Colombia, Universidad de Antioquia, Universidad de Los Andes, Universidad del Valle, Pontificia Universidad Javeriana, Universidad Industrial de Santander, Universidad del Norte, Universidad de Cartagena, Universidad de la Sabana y Universidad Pontificia Bolivariana. Las 
instituciones anteriores comparten como característica la Acreditación de Alta Calidad, reconocimiento del estado colombiano en torno a la calidad de los programas académicos de una institución, su organización, funcionamiento y el cumplimiento de su función social (Consejo Nacional de Acreditación de Colombia, 2017). Adicionalmente, el análisis permite concluir que la Universidad Nacional tiene fuertes lazos de colaboración con las universidades anteriormente mencionadas, y en general, con la mayoría de las universidades del país. Lo anterior, considerando la relación de las principales instituciones colaboradoras (Tabla 2), que se encuentra visible en el perfil de ResearhGate de cada universidad.

Tabla 2: Universidades colombianas con mayor puntaje en RGScore

\begin{tabular}{|c|c|c|c|c|c|c|c|}
\hline \multirow{2}{*}{$\begin{array}{l}\text { Nombre de la } \\
\text { Universidad }\end{array}$} & \multirow{2}{*}{$\begin{array}{c}\text { Acreditaci } \\
\text { ón de alta } \\
\text { calidad }\end{array}$} & \multicolumn{2}{|c|}{$\begin{array}{l}\text { Ubicación } \\
\text { geográfica }\end{array}$} & \multirow{2}{*}{$\begin{array}{l}\text { Número total } \\
\text { de miembros } \\
\text { registrados en } \\
\text { ResearchGate }\end{array}$} & \multirow{2}{*}{$\begin{array}{l}\text { Total RG } \\
\text { Score de la } \\
\text { Universidad } \\
\end{array}$} & \multirow{2}{*}{$\begin{array}{c}\text { Total de } \\
\text { publicaciones } \\
\text { registradas en } \\
\text { ResearchGate }\end{array}$} & \multirow{2}{*}{$\begin{array}{l}\text { Principales instituciones } \\
\text { colaboradoras }\end{array}$} \\
\hline & & $\begin{array}{c}\text { Departa } \\
\text { mento }\end{array}$ & $\begin{array}{l}\text { Muni } \\
\text { cipio }\end{array}$ & & & & \\
\hline $\begin{array}{c}\text { Universidad } \\
\text { Nacional de } \\
\text { Colombia } \\
\end{array}$ & $\mathrm{Si}$ & $\begin{array}{c}\text { Bogotá } \\
\text { D.C }\end{array}$ & $\begin{array}{l}\text { Bogo } \\
\text { tá } \\
\text { D.C. }\end{array}$ & 19520 & 25298,54 & 2973 & $\begin{array}{c}\text { University of Antioquia, } \\
\text { Los Andes University } \\
\text { (Colombia) }\end{array}$ \\
\hline $\begin{array}{l}\text { Universidad } \\
\text { de Antioquia }\end{array}$ & $\mathrm{Si}$ & $\begin{array}{c}\text { Antioqui } \\
\mathrm{a}\end{array}$ & $\begin{array}{l}\text { Med } \\
\text { ellín }\end{array}$ & 5819 & 13729,08 & 2483 & $\begin{array}{l}\text { Hospital Pablo Tobon } \\
\text { Uribe, National } \\
\text { University of Colombia }\end{array}$ \\
\hline $\begin{array}{l}\text { Universidad } \\
\text { de Los Andes }\end{array}$ & $\mathrm{Si}$ & $\begin{array}{l}\text { Bogotá } \\
\text { D.C }\end{array}$ & $\begin{array}{l}\text { Bogo } \\
\text { tá } \\
\text { D.C. }\end{array}$ & 5200 & 7451,86 & 1695 & $\begin{array}{l}\text { Centro Brasileiro de } \\
\text { Pesquisas Físicas, } \\
\text { University of the Andes } \\
\text { (Venezuela) }\end{array}$ \\
\hline $\begin{array}{l}\text { Universidad } \\
\text { del Valle }\end{array}$ & $\mathrm{Si}$ & $\begin{array}{l}\text { Valle } \\
\text { Del } \\
\text { Cauca }\end{array}$ & Cali & 3937 & 6907,07 & 1549 & $\begin{array}{c}\text { Universidad de Jaén, } \\
\text { Hospital Universitario } \\
\text { del Valle }\end{array}$ \\
\hline $\begin{array}{l}\text { Pontificia } \\
\text { Universidad } \\
\text { Javeriana }\end{array}$ & $\mathrm{Si}$ & $\begin{array}{c}\text { Bogotá } \\
\text { D.C }\end{array}$ & $\begin{array}{l}\text { Bogo } \\
\text { tá } \\
\text { D.C. }\end{array}$ & 4958 & 6496,05 & 824 & $\begin{array}{l}\text { National University of } \\
\text { Colombia, Pontificia } \\
\text { Universidad Javeriana - } \\
\text { Cali }\end{array}$ \\
\hline $\begin{array}{l}\text { Universidad } \\
\text { Industrial de } \\
\text { Santander }\end{array}$ & $\mathrm{Si}$ & $\begin{array}{c}\text { Santan } \\
\text { der }\end{array}$ & $\begin{array}{c}\text { Buca } \\
\text { rama } \\
\text { nga }\end{array}$ & 2369 & 4278,94 & 622 & $\begin{array}{l}\text { National University of } \\
\text { Colombia, } \\
\text { University of Santander }\end{array}$ \\
\hline $\begin{array}{l}\text { Universidad } \\
\text { del Norte }\end{array}$ & $\mathrm{Si}$ & $\begin{array}{c}\text { Atlántic } \\
0\end{array}$ & $\begin{array}{c}\text { Barra } \\
\text { nquill } \\
\text { a }\end{array}$ & 1907 & 2948,15 & 172 & $\begin{array}{c}\text { University of South } \\
\text { Florida, National } \\
\text { University of Colombia }\end{array}$ \\
\hline $\begin{array}{l}\text { Universidad } \\
\text { de Cartagena }\end{array}$ & $\mathrm{Si}$ & Bolívar & $\begin{array}{l}\text { Carta } \\
\text { gena }\end{array}$ & 792 & 2473,59 & 1119 & $\begin{array}{c}\text { Universidad Politécnica } \\
\text { de Cartagena, } \\
\text { University of Murcia }\end{array}$ \\
\hline $\begin{array}{l}\text { Universidad } \\
\text { de la Sabana }\end{array}$ & $\mathrm{Si}$ & $\begin{array}{l}\text { Cundin } \\
\text { amarca }\end{array}$ & Chía & 2081 & 2358,51 & 133 & $\begin{array}{l}\text { National University of } \\
\text { Colombia, Pontificia } \\
\text { Universidad Javeriana }\end{array}$ \\
\hline $\begin{array}{l}\text { Universidad } \\
\text { Pontificia } \\
\text { Bolivariana }\end{array}$ & $\mathrm{Si}$ & $\begin{array}{c}\text { Antioqui } \\
\mathrm{a}\end{array}$ & $\begin{array}{l}\text { Med } \\
\text { ellín }\end{array}$ & 1549 & 2353,28 & 281 & $\begin{array}{c}\text { Hospital Pablo Tobon } \\
\text { Uribe, University of } \\
\text { Antioquia }\end{array}$ \\
\hline
\end{tabular}

Para profundizar en los resultados, se realiza la correlación entre el total RGScore frente al número total de miembros registrados en ResearchGate, de las 150 universidades colombianas analizadas en el presente estudio (Tabla 3).

Tabla 3. Correlación entre el total RGScore frente al número total de miembros registrados en ResearchGate

\begin{tabular}{|l|l|l|l|}
\hline \multicolumn{2}{|l|}{} & $\begin{array}{l}\text { Total RGScore de } \\
\text { la Universidad }\end{array}$ & $\begin{array}{l}\text { Número total de miembros } \\
\text { registrados en ResearchGate }\end{array}$ \\
\hline \multirow{2}{*}{\begin{tabular}{l} 
Total RGScore de la $\begin{array}{l}\text { Universidad } \\
\text { Correlación de Pearson }\end{array}$ \\
\cline { 2 - 4 }
\end{tabular}} & 1 &, $973^{* *}$ \\
\cline { 2 - 4 } Sig. La correlación es significativa en el nivel 0,01 (bilateral). &, 000 \\
\cline { 2 - 3 } & N & 150 \\
\hline
\end{tabular}

De acuerdo con los resultados de la tabla 3, el número total de miembros registrados en ResearchGate de la institución, se correlaciona positiva y significativamente con el total RGScore. Este resultado coincide con las conclusiones de los autores Yan et al. (2018), quienes analizan la participación institucional en ResearchGate de las universidades de investigación de Estados Unidos, encontrando que la reputación académica de estas instituciones es directamente proporcional al número usuarios vinculados a ResearchGate. Contrariamente, 
en el contexto de las universidades españolas, González et al. (2015) señalan que no es posible establecer una relación directa entre mayor número de perfiles en ResearchGate y un mayor RGScore. Así, las cuatro universidades españolas con mayor número de perfiles en esta red social son las que tienen mayor número de RGScore, pero a partir del quinto puesto no se sigue la misma tendencia. Así, para que una institución logre un elevado RGScore, "lo que equivale a una mayor reputación científica, no es suficiente con tener un número elevado de perfiles en las redes sociales digitales científicas (RSDC), sino que esos miembros tienen que ser activos: colgar gran número de trabajos, participar en los debates" (González et al., 2015, p. 643). Adicionalmente, los autores González et al. (2015), referente a ResearchGate, considerada la segunda red social científica más utilizada en el mundo, y la primera en universidades españolas, concluye que no hay una relación directa entre mayor número de personal académico y mayor presencia de usuarios en las redes científicas en las universidades.

De otra parte, se analizó el Ranking Top 10 RGScore de las universidades acreditadas en Colombia y el ranking Top 10 RGScore de las universidades no acreditadas en Colombia frente al total RGScore de cada universidad (Figura 1), considerando las instituciones registradas en la base de datos del Sistema Nacional de Información de la Educación Superior (SNIES) (2017).

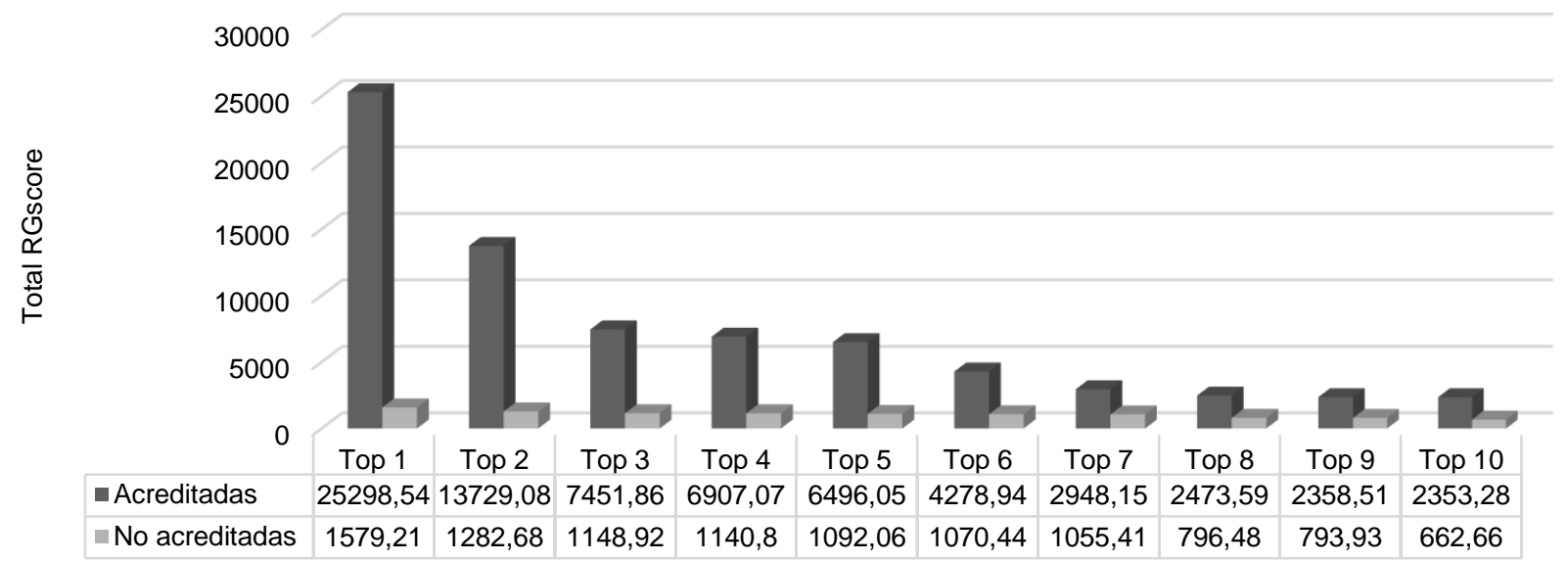

Fig. 1: Ranking Top 10 RGScore de las universidades acreditadas en Colombia Vs Ranking Top 10 RGScore de las universidades no acreditadas en Colombia.

De acuerdo con la Figura 1, de las universidades no acreditadas, las que tienen mayor posicionamiento en ResearchGate son, respectivamente: Universidad Cooperativa de Colombia, Fundación Universitaria Internacional de La Rioja - Unir, Universidad del Atlántico, Universidad Antonio Nariño, Universidad del Quindío, Universidad de Córdoba, Corporación Universidad de la Costa CUC, Universidad Católica de Colombia, Corporación Universitaria Minuto de Dios -Uniminuto-, Universidad de los Llanos. Específicamente, las puntuaciones de RGScore van desde 1579,21 por la Universidad Cooperativa de Colombia a 662,66 por la Universidad de los Llanos. Considerando los resultados de la Figura 1 se evidencian significativas diferencias en el RGScore de las universidades acreditadas y no acreditadas. Específicamente, las primeras universidades colombianas con mejor posicionamiento en ResearchGate correspondientes a la Universidad Nacional, Universidad de Antioquia y Universidad de los Andes. Lo anterior, da cuenta de la importancia de los procesos de acreditación institucional para consolidar capacidades en materia investigativa y productividad científica. De la misma manera, al comparar los resultados en materia de publicaciones registradas en ResearchGate (Figura 2), se evidencian diferencias significativas entre las universidades acreditadas y no acreditadas.

El análisis de los resultados, presentan una correlación entre el total RGScore de la institución frente al número total de publicaciones registradas en ResearchGate, de las 150 universidades colombianas analizadas en el presente estudio (Tabla 4).

Tabla 4. Correlación entre el total RGScore frente al número total de publicaciones registradas en ResearchGate

\begin{tabular}{|l|l|l|l|}
\hline \multicolumn{2}{|c|}{} & $\begin{array}{l}\text { Total RGScore de } \\
\text { la Universidad }\end{array}$ & $\begin{array}{l}\text { Total Publicaciones registradas } \\
\text { en ResearchGate }\end{array}$ \\
\hline \multirow{2}{*}{$\begin{array}{l}\text { Total RGScore de la } \\
\text { Universidad }\end{array}$} & Correlación de Pearson & 1 &, $924^{* *}$ \\
\cline { 2 - 4 } & Sig. (bilateral) & &, 000 \\
\cline { 2 - 4 } & $\mathrm{N}$ & 150 & 118 \\
\hline \multirow{2}{*}{ **. La correlación es significativa en el nivel 0,01 (bilateral). } \\
\hline
\end{tabular}




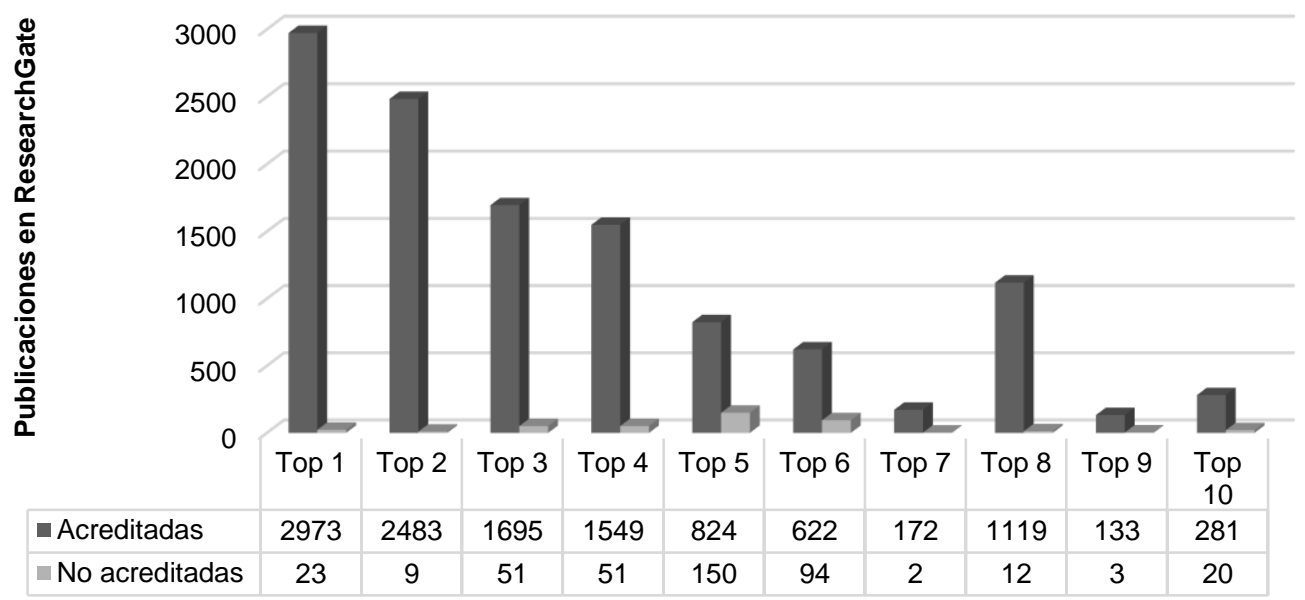

Fig. 2: Comparación del total de publicaciones registradas en ResearchGate frente al ranking Top 10 RGScore de las universidades acreditadas y no acreditadas en Colombia.

De acuerdo con la estadística presentada en la tabla 4, el número total de publicaciones registradas en ResearchGate de la institución, se correlaciona positiva y significativamente con el total RGScore. En este sentido, la literatura destaca que el número de veces que un artículo ha sido leído en ResearchGate tiene una correlación positiva con su recuento de citas Scopus, confirmando que los indicadores de ResearchGate pueden ser significativos (Thelwall y Kousha, 2017). En contraste, los estudios de Thelwall y Kousha (2017) señalan que ResearchGate se correlacionó más fuertemente con las citas de Google Scholar, lo que sugiere que ResearchGate no utiliza predominantemente una fuente de datos fundamentalmente diferente a Google Scholar; sin embargo, el intercambio de preimpresiones en ResearchGate es lo suficientemente importante como para que los autores lo tomen en serio. Pese a lo anterior, la aceptación de ResearchGate varía mucho a escala internacional (Thelwall y Kousha, 2015) y, por lo tanto, es probable que sus datos contengan algunos sesgos sistemáticos. Además, puede indexar en sus indicadores productos de baja calidad (Memon, 2016).

Puntualmente, en el caso de Latinoamérica, la literatura destaca diferencias entre la visibilidad de los investigadores en ResearchGate frente a la reportada por sistemas nacionales de medición de ciencia, tecnología e innovación. A manera de ejemplo, investigaciones desarrolladas en el contexto colombiano destacan que "al comparar la información de GS (Google Scholar) y RG (ResearchGate) con las cuatro categorías de CvLAC (Colciencias ScienTI) se observa que un porcentaje significativo de investigadores que aparecen clasificados como Reconocidos o Junior muestran una importante visibilidad web e impacto. De forma similar, otro alto porcentaje de los categorizados como Sénior o Asociado presentan valores bajos en GS y RG incluso en casos donde su producción científica de acuerdo a CvLAC es muy alta" (Aguillo et al., 2017). En el mismo sentido, Campos et al. (2014) destacan la carencia de una estrategia institucional proactiva, lo cual reviste complejidad en el caso de ResearchGate debido a que la iniciativa de la afiliación e interacción corresponde a los propios académicos, por lo cual el ranking de ResearchGate no coincide en la mayoría de los casos con las categorizaciones definidas por los sistemas públicos de evaluación en el caso de Ecuador, ni con otras clasificaciones reconocidas de las universidades de Colombia y Perú.

Adicionalmente, los resultados de Yan y Zhang (2018) en 61 universidades de investigación en Estados Unidos confirman que ResearchGate es un sitio de redes sociales académicas orientado a la investigación que refleja de cerca y de manera realista el nivel de actividad de la investigación de las instituciones. Puntualmente, el estudio de Borrego (2017) en el contexto de las universidades españolas, destaca que ResearchGate cuenta con mayor número de documentos alojadas que los repositorios institucionales. Igualmente, el estudio de Batooli (2017) destaca que en el contexto de los investigadores iraníes el coeficiente de correlación entre las variables de visualización, descarga y el número de citas de los artículos en ResearchGate y el número de citas de los artículos en Science Citation Index (SCI) fueron positivos e importantes.

En otro orden de ideas, en el presente artículo se analiza el total RGScore de las Universidades de Bogotá, distrito capital de Colombia, el cual concentra un número importante de universidades, frente al total RGScore de las universidades de Colombia exceptuando a Bogotá (Figura 3). En este sentido, las universidades con mayor puntuación en el RGScore ubicadas en Bogotá son, en su orden: Universidad Nacional de Colombia, Universidad de los Andes, Pontificia Universidad Javeriana, Universidad Militar-Nueva Granada, Universidad El Bosque, Universidad Distrital-Francisco José de Caldas, Universidad de la Salle, Fundación Universitaria Internacional de La Rioja - UNIR, Fundación Universidad de Bogotá - Jorge Tadeo Lozano y la Universidad Antonio Nariño. En contraste, las universidades del país mayor posicionamiento en el RGScore, con sede 
principal en ciudades diferentes a Bogotá son: Universidad de Antioquia (Medellín), Universidad del Valle (Cali), Universidad Industrial de Santander (Bucaramanga), Universidad del Norte (Barranquilla), Universidad de Cartagena (Cartagena), Universidad de la Sabana (Chía), Universidad Pontificia Bolivariana (Medellín), Universidad Pedagógica y Tecnológica de Colombia - UPTC (Tunja), Universidad del Cauca (Popayán) y la Universidad de Caldas (Manizales).

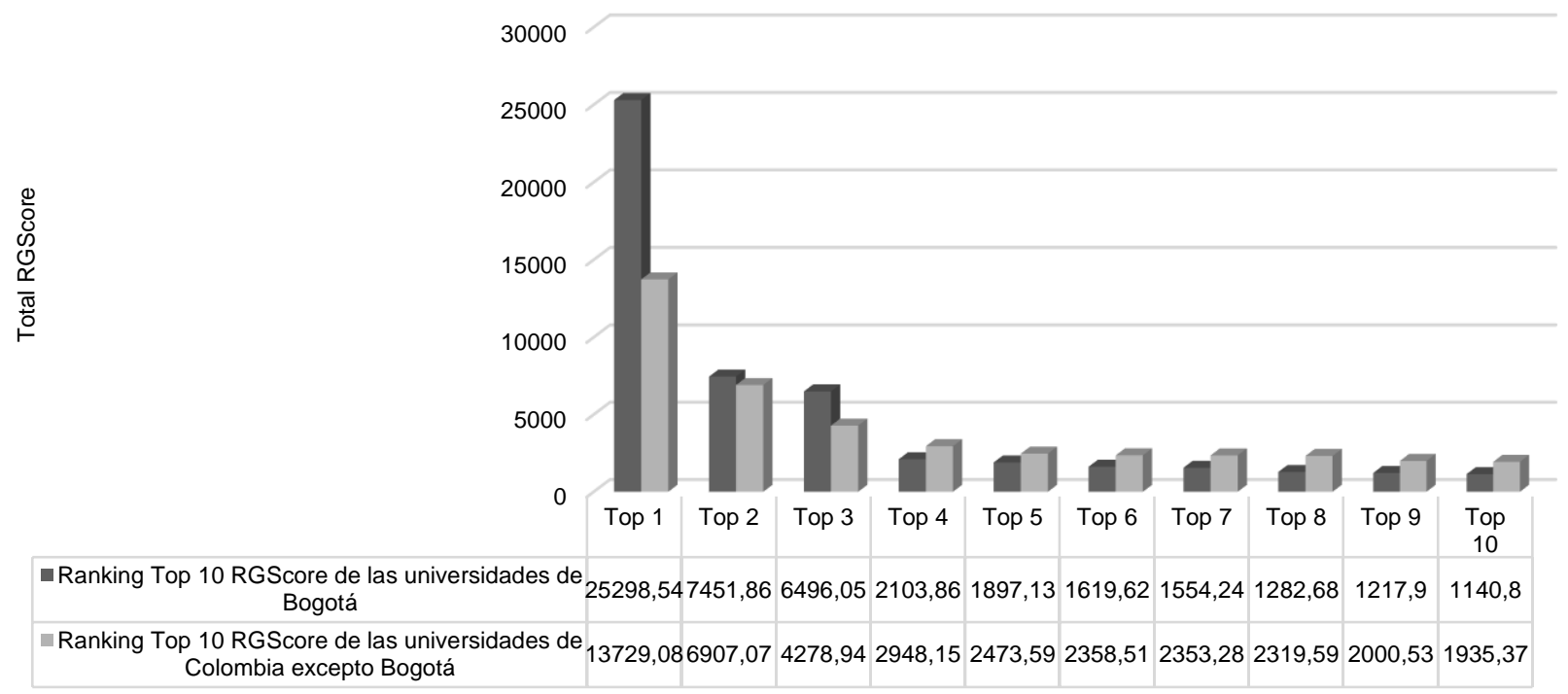

Fig. 3: Ranking Top 10 RGScore de las universidades de Bogotá Vs Ranking Top 10 RGScore de las universidades de Colombia excepto Bogotá

De acuerdo con la figura 3, al analizar el RGScore de las universidades de Bogotá frente al ranking top 10 RGScore de las universidades colombianas excepto Bogotá se evidencia que las diferencias son menos significativas que al comparar las universidades acreditadas frente a las no acreditadas; lo que implica que el factor geográfico no es determinante en materia de productividad científica en Colombia. Lo anterior, se corrobora en la tabla 5 y la figura 4.

Tabla 5: Número de Grupos de Investigación, Desarrollo Tecnológico e Innovación clasificados y reconocidos por Colciencias por departamento y el promedio RGScore de las universidades del departamento

\begin{tabular}{|c|l|c|c|}
\hline$N^{\circ}$ & $\begin{array}{l}\text { Departamento de } \\
\text { Colombia }\end{array}$ & $\begin{array}{c}\text { Número de Grupos de } \\
\text { Investigación, Desarrollo } \\
\text { Tecnológico e Innovación } \\
\text { clasificados y reconocidos por } \\
\text { Colciencias por departamento }\end{array}$ & $\begin{array}{c}\text { Promedio RGScore de las } \\
\text { Universidades por } \\
\text { departamento }\end{array}$ \\
\hline 1 & Bogotá D.C & 1617 & 1094,24 \\
\hline 2 & Antioquia & 716 & 995,00 \\
\hline 3 & Valle del Cauca & 386 & 987,14 \\
\hline 4 & Santander & 230 & 716,46 \\
\hline 5 & Atlántico & 214 & 886,30 \\
\hline 6 & Bolívar & 169 & 581,83 \\
\hline 7 & Caldas & 144 & 637,21 \\
\hline 8 & Boyacá & 134 & 975,75 \\
\hline 9 & Risaralda & 118 & 676,48 \\
\hline 10 & Nariño & 93 & 393,68 \\
\hline 11 & Norte de Santander & 89 & 388,63 \\
\hline 12 & Cundinamarca & 88 & 1238,49 \\
\hline 13 & Cauca & 79 & 516,57 \\
\hline 14 & Tolima & 71 & 154,61 \\
\hline 15 & Magdalena & 63 & 834,64 \\
\hline 16 & Córdoba & 61 & 644,39 \\
\hline 17 & Quindío & 57 & 548,96 \\
\hline 18 & Huila & 57 & 279,91 \\
\hline
\end{tabular}


Tabla 5 (continuación)

\begin{tabular}{|c|l|c|c|}
\hline 19 & Meta & 50 & 337,83 \\
\hline 20 & La Guajira & 37 & 234,02 \\
\hline 21 & Sucre & 36 & 262,45 \\
\hline 22 & Cesar & 34 & 141,79 \\
\hline 23 & Caquetá & 23 & 229,64 \\
\hline 24 & Chocó & 14 & 60,39 \\
\hline
\end{tabular}

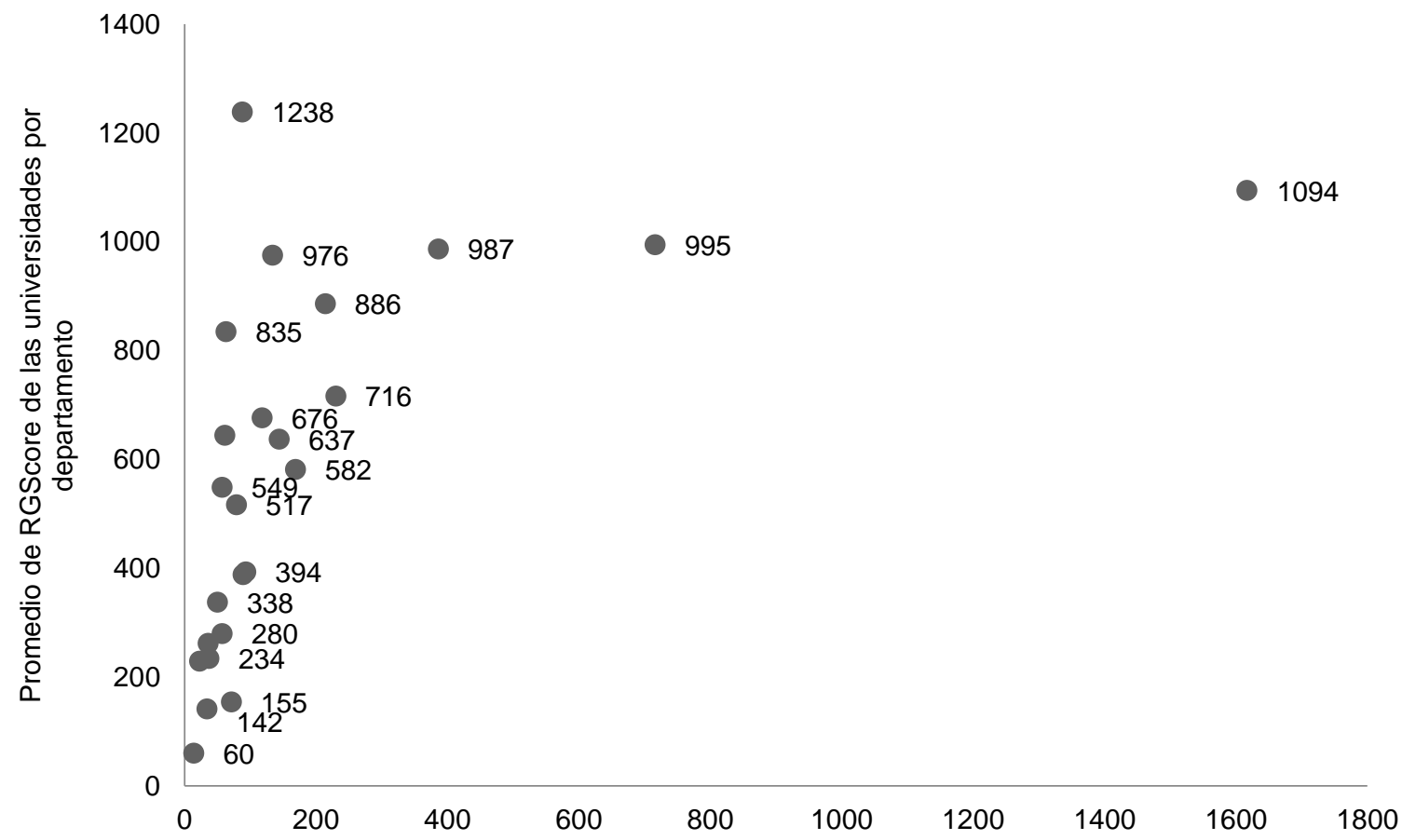

Nº de Grupos de Investigación, desarrollo tecnológico e innovación clasificados y reconocidos por COLCIENCIAS por departamento

Fig. 4: Relación entre el número de Grupos de Investigación, Desarrollo Tecnológico e Innovación clasificados y reconocidos por Colciencias por departamento y el Promedio RGScore de las Universidades por departamento.

Adicionalmente, los planteamientos anteriores se corroboran con la correlación entre el número de grupos por departamento y promedio RGScore de las universidades por departamento (Figura 4), en el cual, mediante la correlación de Pearson, se obtiene un coeficiente de 0.53 (Tabla 6), lo que indica la existencia de una correlación lineal positiva débil. Lo anterior implica que, si bien los departamentos con mayor cantidad de grupos de investigación tienen mayor visibilidad de los investigadores en ResearchGate, esto no representa una tendencia generalizada, denotando la existencia de departamentos con pocos grupos de investigación y altos niveles de visibilidad en ResearchGate.

Tabla 6: Correlación entre el número de grupos por departamento y el promedio RGScore de las universidades por departamento

\begin{tabular}{|c|l|c|c|}
\hline \multicolumn{2}{|c|}{} & $\begin{array}{c}N^{\circ} \text { de grupos por } \\
\text { departamento }\end{array}$ & $\begin{array}{c}\text { Promedio RGScore de las } \\
\text { Universidades por departamento }\end{array}$ \\
\hline \multirow{4}{*}{\begin{tabular}{c}
$N^{*} \begin{array}{c}\text { No grupos por } \\
\text { departamento }\end{array}$ \\
\cline { 2 - 4 }
\end{tabular}} & Correlación de Pearson & $\begin{array}{l}\text { Suma de cuadrados y productos } \\
\text { vectoriales }\end{array}$ & $26394^{* *}$ \\
\cline { 2 - 4 } & Covarianza & 114740,31 &, 007 \\
\cline { 2 - 4 } & $\mathrm{N}$ & 24 & 1398391,42 \\
\hline
\end{tabular}




\section{CONCLUSIONES}

A partir de los resultados obtenidos se concluye: las universidades colombianas con mayor RGScore son: la Universidad Nacional de Colombia, la Universidad de Antioquia, la Universidad de los Andes, la Universidad del Valle y la Pontificia Universidad Javeriana. Las 10 universidades colombianas con mayor puntaje total en el RGScore corresponden a universidades con acreditaciones en alta calidad. Producto del análisis realizado a los indicadores de las 150 universidades colombianas registradas en ResearchGate, se concluye que existe una correlación significativa y positiva entre el total RG de la Universidad y el número de miembros registrados en ResearchGate. En el mismo sentido, los indicadores del puntaje total de RGScore y el número de publicaciones se correlacionan positivamente y significativamente en las universidades analizadas. De otro lado, existen diferencias significativas en el puntaje total de RGScore y en el número de publicaciones registradas en ResearchGate al comparar el ranking top 10 de las universidades acreditadas frente a las no acreditadas en Colombia. Finalmente, se concluye la existencia de una correlación positiva débil entre el número de grupos por departamento y promedio RGScore de las Universidades por departamento, indicando que, si bien, los departamentos con mayor cantidad de grupos de investigación tienen mayor visibilidad de los investigadores en ResearchGate, esto no representa una tendencia generalizada en el caso colombiano.

\section{REFERENCIAS}

Aguillo, I.F., A. Uribe y W. López, Visibilidad de los investigadores colombianos según sus indicadores en Google Scholar y ResearchGate. Diferencias y similitudes con la clasificación oficial del sistema nacional de ciencia - COLCIENCIAS, doi: 10.17533/udea.rib.v40n3a03, Rev. Interam. Bibliot., 40(3), 221-230 (2017)

Araujo, R., L. Oliveira y C. Furtado, A rede social acadêmica researchgate como mecanismo de visibilidade e internacionalização da produção científica brasileira e portuguesa na área de Biblioteconomia e Ciência da Informação, doi:dx.doi.org/10.1590/1981-5344/2937, Perspectivas em Ciência da Informação, 22(4), 177-207 (2017)

Batooli, Z., The relationship between Web of Science and ResearchGATE Indicators of Iranian researchers' Top Papers, Iranian Journal of Information Processing Management, 33(1), 163-186 (2017)

Bhardwaj, R., Academic social networking sites: Comparative analysis of ResearchGate, Academia.edu, Mendeley and Zotero, doi:10.1108/ILS-03-2017-0012, Information and Learning Science, 118(5-6), 298-316 (2017)

Borrego, Á., Institutional repositories versus ResearchGate: The depositing habits of Spanish researchers, doi:10.1002/leap.1099, Learned Publishing, 30(3), 185-192 (2017)

Campos, F., D. Rivera y C. Rodríguez, La presencia e impacto de las universidades de los países andinos en las redes sociales digitales, ISSN: 1138-582, Revista Latina de Comunicación Social, 69, 571 - 592 (2014)

Consejo Nacional de Acreditación de Colombia, ¿Qué es la Acreditación?, M. d. Nacional, Productor (2017)

Departamento Administrativo de Ciencia, Tecnología e Innovación - COLCIENCIAS, Modelo de Medición de Grupos de Investigación, Desarrollo Tecnológico o de Innovación y de Reconocimiento de Investigadores del Sistema Nacional de Ciencia, Tecnología e Innovación, Año 2017, 04 (2017)

Departamento Administrativo de Ciencia, Tecnología e Innovación - COLCIENCIAS, Plataforma SCIENTI. (2017)

Galligan, F. y S. Dyas Correia, Altmetrics: rethinking the way we measure, ISSN: 1879-095X, Serials Review, 39(1), 56$61(2013)$

González, C., M. Iglesias y L. Codina, Presencia de las Universidades españolas en las redes sociales digitales científicas: casos de los estudios de comunicación, doi:dx.doi.org/10.3145/epi.2015.sep.12, El profesional de la información, 24(5) (2015)

Jamali, H., Copyright compliance and infringement in ResearchGate full-text journal articles, doi:10.1007/s11192-0172291-4, Scientometrics, 112, 241-254 (2017)

Manca, S., ResearchGate and Academia.edu as networked socio-technical systems for scholarly communication: a literature review, doi:dx.doi.org/10.25304/rlt.v26.2008, Research in Learning Technology, 26 (2018)

Mangkhollen, S. y A. Mohammad, Use of ResearchGate by the Research Scholars of Pondicherry University: A Study, doi:10.14429/djlit.37.11139, Journal of Library \& Information Technology, 37(5), 366-371 (2017)

Memon, A.R., ResearchGate is no longer reliable: Leniency towards ghost journals may decrease its impact on the scientific community, Journal of the Pakistan Medical Association, 66(12), 1643-1647 (2016)

Míguez, M., I. Puentes y A. Dafonte, Academic social networks and communication researchers from universities in the north of Portugal: An analysis of Academia.edu and ResearchGate, doi:10.1007/978-3-319-46068-0_53, Advances in Intelligent Systems and Computing, 503, 405-411 (2017)

Muscanell, N. y S. Utz, Social networking for scientists: An analysis on how and why academics use ResearchGate, doi:10.1108/OIR-07-2016-0185, Online Information Review, 41(5), 744-759 (2017)

Orduna, E., A. Martín. M. Thelwall y E. López, Do ResearchGate Scores create ghost academic reputations?, doi:10.1007/s11192-017-2396-9, Scientometrics, 112, 443-460 (2017) 
Orduña, E., A. Martín y E. Delgador, RESEARCHGATE como fuente de evaluación científica: desvelando sus aplicaciones bibliométricas, doi:dx.doi.org/10.3145/epi.2016.mar.18, El profesional de la información, 25(2) (2016)

Ortega, J.L., Redes sociales académicas ¿espacios colaborativos o clubes Diógenes?, doi:doi.org/10.3145/thinkepi.2017.42, Anuario ThinkEPI, (1), 225-229 (2017)

Puentes, I., S. Direito y D. Lago, Las redes sociales científicas: presencia y actividad de los investigadores de comunicación de las universidades de Portugal en Academia.edu y ResearchGate.net, doi:10.4185/cac90, Universidad de La Laguna, 2830-2846 (2015)

Punín, M.I., D.R. Sabela y D. Calva, La participación e interacción de los investigadores de comunicación de países iberoamericanos en las redes sociales digitales científicas, Congreso Internacional Latina de Comunicación, La Laguna Tenerife (2014)

ResearchGate. ResearchGate, Share and discover research (2017)

Robinson, N., D. Torres, Z. Zahedi y R. Costas, New data, new possibilities: Exploring the insides of Altmetric.com, El profesional de la información, 23(04), 359-366 (2014)

Santana, S., Redes de intercambio de información científica y académica entre los profesionales en el contexto de la Web 2.0., Revista Cubana de ACIMED, 21(3), 321-333 (2010)

Singson, M. y M. Amees, Use of ResearchGate by the Research Scholars of Pondicherry University: A Study, doi:10.14429/djlit.37.11139, Journal of Library \& Information Technology, 37(5), 366-371 (2017)

Sistema Nacional de Información de la Educación Superior SNIES, Busqueda de Instituciones de Educación Superior Aprobadas, M. d. Nacional, Productor (2017)

Thelwall, M. y K. Kousha, ResearchGate: Disseminating, communicating and measuring scholarship?, doi:10.1002/asi.23236, Journal of the Association for Information Science and Technology, 66(5), 876-889 (2015)

Thelwall, M. y K. Kousha, ResearchGate articles: Age, discipline, audience size and impact, doi:10.1002/asi.23675, Journal of the Association for Information Science and Technology, 68(2), 468-479 (2017)

Thelwall, M. y K. Kousha, ResearchGate versus Google Scholar: Which finds more early citations?, doi:doi.org/10.1007/s11192-017-2400-4, Scientometrics, 112, 1125-1131 (2017)

Yan, W. e Y. Zhang, Research universities on the ResearchGate social networking site: An examination of institutional differences, research activity level, and social networks formed, doi:10.1016/j.joi.2017.08.002, Journal of Informetrics, 12(1), 385-400 (2018)

Yan, W., Y. Zhang y W. Bromfield, Analyzing the follower-followee ratio to determine user characteristics and institutional participation differences among research universities on ResearchGate, doi:doi.org/10.1007/s11192-018-2637-6, Scientometrics, 115, 299-316 (2018)

Yu, M.C., Y.C. Wu y otros 3 autores, ResearchGate: An effective altmetric indicator for active researchers?, doi:doi.org/10.1016/j.chb.2015.11.007, Computers in Human Behavior, 55, 1001-1006 (2016) 
